\title{
Maxillary Canine-to-First Premolar Bilateral Transposition in a Female with Down Syndrome
}

\author{
A Case Report \\ Sabiha A. Al-Mutawa ${ }^{a}$ Maddi Shyama ${ }^{a}$ Eino Honkalab \\ a Oral Health Services, Ministry of Health, and ${ }^{\text {b} F a c u l t y ~ o f ~ D e n t i s t r y, ~ U n i v e r s i t y ~ o f ~ K u w a i t, ~ K u w a i t ~}$
}

\section{Key Words}

Transposition, maxillary canine to first premolar · Down syndrome

\begin{abstract}
Objective and Importance: To report a case of the bilateral transposition of the maxillary canines with the premolars in a 17-year-old female with Down syndrome. Clinical Presentation and Intervention: A 17-year-old female presented with moderate mental retardation; she was short and of small stature, and she had a round cherubic face and a broad forehead. Clinical intraoral examination revealed bilateral malpositions of the maxillary canines and premolars as transposition. She had an open mouth, macroglossia and a protruding tongue. The case required the combined orthodontic and prosthetic treatment to achieve an acceptable morphologic and esthetic occlusion. However, the parents refused to accept the proposed treatment. Extractions of the roots and caries treatment were done as these were considered priority for the patient. Conclusion: This rare and severe positional anomaly represents an orthodontic challenge and its correction involves treatment risk and requires a great deal of control and carefully applied mechanics.
\end{abstract}

Copyright (C) 2003 S. Karger AG, Basel

\section{KARGER}

Fax +41613061234

E-Mail karger@karger.ch

www.karger.com
(C) 2003 S. Karger AG, Basel

1011-7571/03/0123-0193\$19.50/0

Accessible online at:

www. karger.com/mpp

\section{Introduction}

Transposition of teeth is a rare and uncommon dental anomaly of unknown causes or uncertain origin and affects $0.4 \%$ of the population $[1,2]$. Tooth transposition presents a major diagnostic and therapeutic challenge in the correction of the malocclusion. Transposition of teeth has been defined as the positional interchange of two adjacent teeth with ectopic eruption of permanent teeth characterized by the development of the eruption of a tooth into a position occupied normally by a nonadjacent tooth [1-3]. Dental transposition is a developmental alteration resulting in a deviation in tooth position, clinically identified as the interchange of two adjacent teeth that alters the natural order of the dental arch [1-4]. Transposition is often accompanied by other dental anomalies, such as congenitally missing or peg-shaped maxillary lateral incisors, severe rotations and malpositions of the adjacent teeth, retained deciduous teeth, dilacerations and malformation of other teeth or rotation of the transposed teeth [5]. Transpositions may occur unilaterally or bilaterally and their gender ratio varies according to type. Unilateral transpositions are much more prevalent than bilateral ones [6]. Transposition of teeth may occur both in the maxillary and mandibular arches, but it occurs 


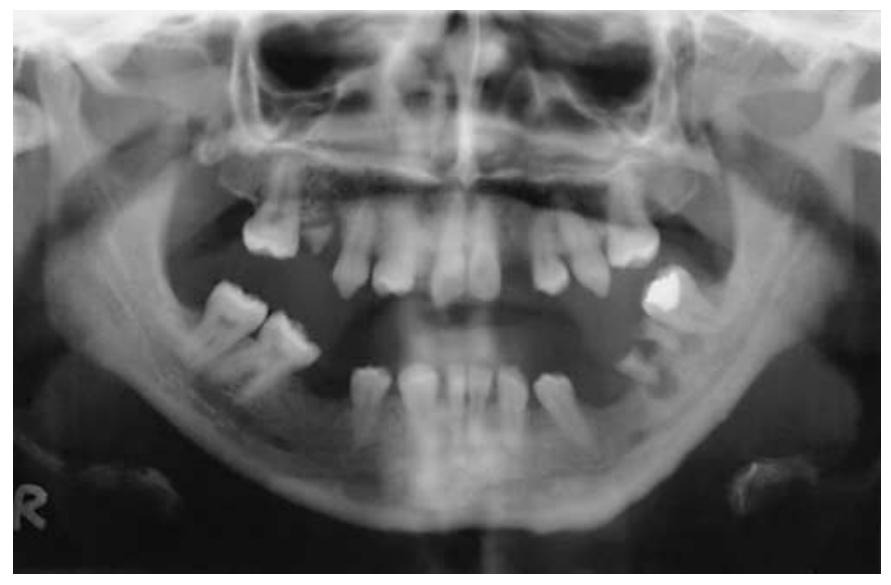

Fig. 1. Panoramic radiograph showing complete bilateral transposition of maxillary canines with the premolars and partial anodontia.

more often in the maxilla [5]. The maxillary permanent canine shows the highest incidence of transposition, changing its eruptive place with the first premolar in most cases [3, 7], less often with the lateral incisor [8], rarely with the central incisor [9] and very rarely with the second premolar [10] or the first molar [11].

The maxillary canine-to-first premolar transposition was determined to be an anomaly resulting from genetic influences within a multifactorial inheritance model [3]. A study by Peck et al. [3] found evidence to suggest that the maxillary canine-to-first premolar transposition is genetically influenced due to the moderate rate of bilateral occurrence, sex-associated frequency differences, increased prevalence of additional dental anomalies, and occurrence along family lines. In some cases the canineto-first premolar transposition occurs simultaneously with developmentally absent lateral incisors [3, 4] and is the most frequently appearing maxillary transposition type, comprising $71 \%$ of the cases [4].

While bilateral maxillary canine-to-first premolar transposition is a rare condition and requires complex orthodontic and/or prosthetic treatment, this case is reported to the medical and dental clinicians so that they can recognize the need for early detection of transpositions, planning and provision of an appropriate treatment.

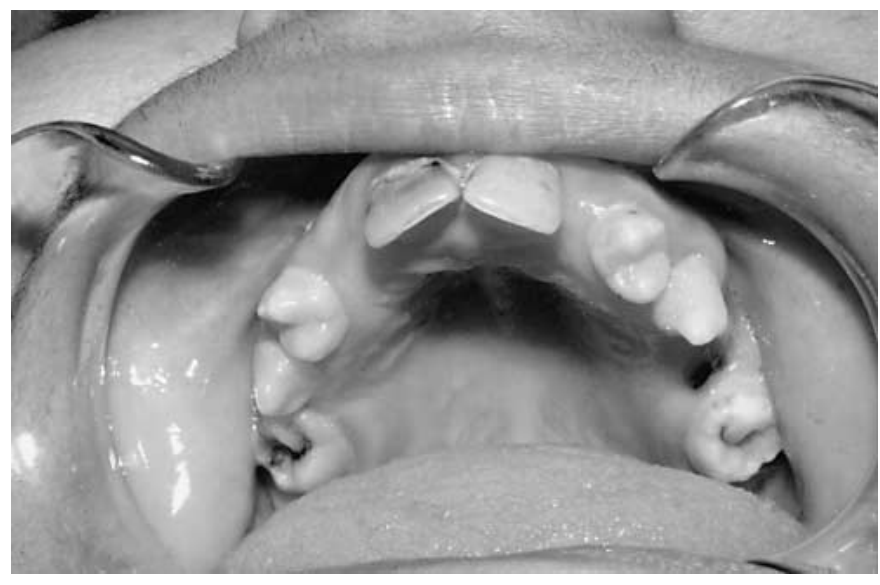

Fig. 2. Intraoral view showing transposed maxillary canines and first premolars.

\section{Case Report}

A 17-year-old female was examined in the special-needs school of Down syndrome girls in Kuwait for a routine dental checkup. Past medical history revealed her mental disability diagnosed as Down syndrome. Psychological evaluation showed her IQ to be 40 (moderate mental retardation). She had a history of delayed speech and developmental milestones.

On general examination, she appeared short and of small stature. Hands, feet and digits were broad and shortened. Her weight was $53 \mathrm{~kg}$ and height $140 \mathrm{~cm}$. The skin of the facial area appeared dry. Extraoral examination revealed a round cherubic face, broad forehead, microcephaly, slanting eyes, flat nasal bridge, flattening of the occiput, and a broad and short neck.

The clinical intraoral examination revealed the bilateral malpositions of the maxillary canines and premolars as transposition. She had an open mouth, macroglossia and a protruding tongue. She had a tongue-thrust habit. The following teeth were present: $16,55,14,13$, $11,21,23,24,26,37,36,34,33,31,41,43,44,46$, and 47 (fig. 1). The following teeth were developmentally absent: lateral incisors and second premolars in the maxillary (fig. 1,2) and mandibular arches and the second molars in the maxilla. The following teeth had caries: $16,55,11,21,26,36,46$, and 47 . Teeth 55 and 36 had remaining root stumps. Only one tooth, 37 , had been restored. Her oral hygiene was poor. The maxillary arch was narrow and the mandibular arch broad, forming bilateral posterior crossbite in occlusion (fig. 3). The palatal vault was narrow and short. In addition to complete bilateral transposition of $13 / 14$ and 23/24, the orthodontic diagnosis was: bilateral crossbite, large edentulous spaces in both arches, small midline deviation, but acceptable overjet and overbite.

The case required a combined orthodontic and prosthetic treatment to achieve an acceptable morphologic and esthetic occlusion. The suggested orthodontic treatment was: maxillary expansion and slight mandibular constriction to correct the crossbite; opening ideal pontic spaces for prosthetic replacement of 12 and 22; maintaining the maxillary tooth positions by placing 14 and 24 in the positions of 13 and 23 and placing 13 and 23 in the positions of 14 and 24; open- 
ing ideal pontic spaces for prosthetic replacement of 15 and 25; placing 33 and 43 in the positions of 32 and 42 ; placing 34 and 44 in the positions of 33 and 43 and opening ideal pontic spaces for prosthetic replacement of $36,35,34,44,45$. The suggested prosthodontic treatment was: conventional bridges or implant restorations for replacement of the edentulous areas 15, 12, 22, 25, 36, 35, 34, 44, 45. Caries treatment was considered as a priority for this patient. Oral prophylaxis was done and the carious teeth was restored. The patient was given oral hygiene instructions and dietary counseling. Preventive measures were undertaken in view of the high caries rate. The root stumps of 36 and 55 were extracted. However, orthodontic treatment was not done because the family did not yet accept the treatment. It is hoped that orthodontic treatment will be possible at a later stage.

\section{Discussion}

Although the etiology of tooth transposition is unknown, several theories have been suggested to explain the causes and development of transposed teeth and these include: early loss of deciduous canines, crowding, and heredity [6]. Transposition in the maxilla is a result of displacement and migration of the maxillary canine. The long path of eruption is a common cause of maxillary canine displacement, impaction, and transposition [12]. The great migratory ability of the canine might be the reason that it is the tooth most commonly involved in transpositions.

Congenitally missing teeth are commonly seen with Down syndrome and mentally retarded subjects [13-15]. McMillin et al. [13] and Tannenbaum and Miller [14] have observed 35 and $34 \%$, respectively, of congenitally missing teeth in 174 institutionalized individuals with Down syndrome, and in 38 institutionalized retarded patients. An increased incidence of missing primary lateral incisors in 122 Down syndrome children was noted by Barkla [15]. No reports are available on the transposition of teeth among Down syndrome subjects; the transposition might not be related to Down syndrome and could be merely a coincidence.

There are different opinions about reversing a transposition. Early treatment has been recommended, because the treatment can be accomplished with fewer possibilities of injuring the soft tissues [8]. Various treatment options have been proposed for bilaterally transposed teeth, including alignment in the transposed positions, extraction of one or both transposed teeth, and orthodontic movement into the normal arch positions [8]. Orthodontic treatment planning centers around the extractionnonextraction decision and the question of correcting the transposed tooth order. Maxillary canine-to-first premolar transposition is usually best managed orthodontically

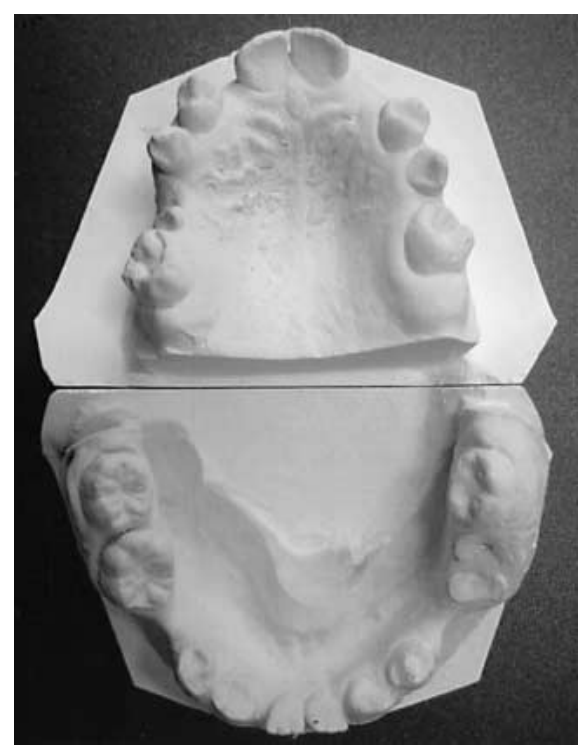

Fig. 3. The maxillary and mandibular arches in occlusal view.

on a nonextraction basis, keeping the transposed order of the teeth. In some cases the canine-to-first premolar transposition occurs simultaneously with the developmentally absent lateral incisors. In a spaced arch, the transposition should be accepted and the space localized to the lateral incisor region. A fixed, removable, or an implant prosthesis may then be considered for its replacement. The clinician must be aware of the esthetics, occlusion, cuspid root apex position, treatment length, patient cooperation, periodontal support, and patient's age when correcting transposed teeth.

\section{Conclusion}

This rare and severe positional anomaly represents an orthodontic challenge and its correction involves treatment risk and requires a great deal of control and carefully applied mechanics. 


\section{References}

1 Maia FA: Orthodontic correction of a transposed maxillary canine and lateral incisor. Angle Orthod 2000;70:339-348.

2 Chattopadhyay A, Srinivas K: Transposition of teeth and genetic etiology. Angle Orthod 1998; 66:147-152.

3 Peck L, Peck S, Attia Y: Maxillary canine-first premolar transposition, associated dental anomalies and genetic basis. Angle Orthod 1993;63:99-109.

4 Peck S, Peck L: Classification of maxillary tooth transpositions. Am J Orthod Dentofacial Orthop 1995;107:505-517.

5 Shapira Y: Transposition of canines. J Am Dent Assoc 1980;100:710-712.
6 Weeks EC, Power SM: The presentations and management of transposed teeth. Br Dent J 1996;18:421-424.

7 Burnett SE: Prevalence of maxillary caninefirst premolar transposition in a composite African sample. Angle Orthod 1999;69:187189.

8 Shapira Y, Kuftinec M: Maxillary canine-lateral incisor transposition: Orthodontic management. Am J Orthod 1989;95:439-444.

9 Jackson M: Upper canine in position of upper central incisor. Br Dent J 1951;90:243.

10 Joshi MR, Gaitonde SS: Canine transposition of extensive degree: A case report. Br Dent $\mathbf{J}$ 1966;121:221-222.
11 Hallett GE: A maxillary canine erupting in the first molar region. Br Dent J 1942;72:191.

12 Mitchell L: Canines; in Mitchell L (ed): An Introduction to Orthodontics. Oxford, Oxford University Press, 1996, p 137.

13 McMillin RS, Kashgarian M: Relation of human abnormalities of structures and functions of the dentition. II. Mongolism. J Am Dent Assoc 1961;63:368-373.

14 Tannenbaum KA, Miller JW: Oral conditions of the mentally retarded patient. J Dent Child 1961;28:277-280.

15 Barkla DH: Congenital absence and fusion in the deciduous dentition. J Ment Defic Res 1963;7:102-106. 